\title{
European Addiction Research: A New Editor, a New Advisory Board and Intriguing Perspectives
}

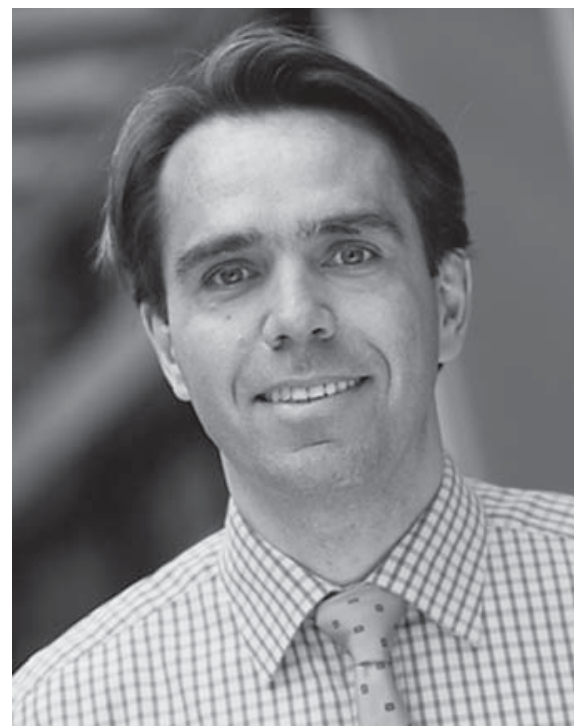

Falk Kiefer

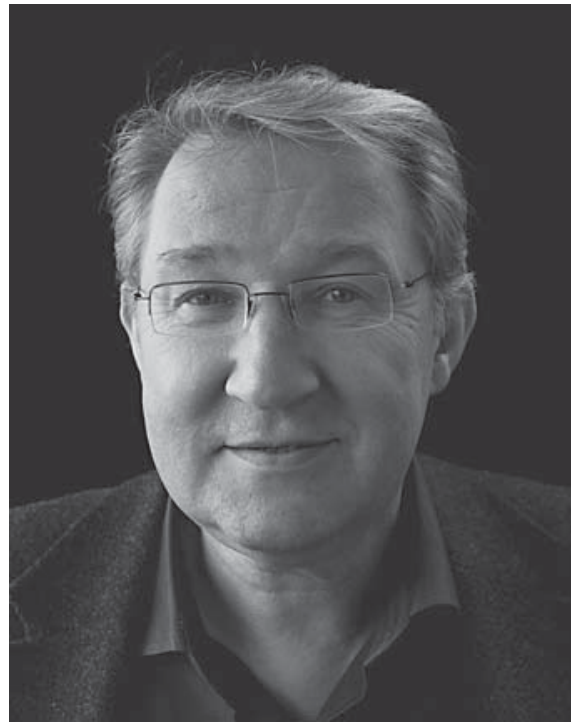

Wim van den Brink
European addiction research is developing rapidly and accumulating innovative research requires consolidation of an interdisciplinary forum for the exchange and discussion of recent results and consequences, reflecting the importance of a comprehensive approach to resolve the problems of substance abuse and addiction in Europe.

Experimental and recreational alcohol, tobacco and drug use as well as excessive exposure to 'nonsubstance reinforcers' like gambling are important phenomena that are biopsychosocially determined and carry the risk of out-of-control use, harmful use, dependence, and even chronic addiction. European institutions increasingly recognize the problem of the enormous costs associated with addictive behaviors. About 37 million Europeans are estimated to suffer from substance dependence resulting in an annual 110 billion Euros of societal costs, including heath care, unemployment, and crime-related costs (European Brain Council, 2005).

From a health economic perspective, two facts are of special relevance: First, 'legal drugs', i.e. alcohol and nic-

\section{KARGER}

Fax +41 613061234 E-Mail karger@karger.ch www.karger.com (c) 2011 S. Karger AG, Basel

1022-6877/11/0172-000I\$38.00/0 
otine, are responsible for the majority of this burden and, therefore, the strategy to tackle alcohol- and nicotine-related problems predominantly with campaigns on prevention, while targeting treatment studies mainly towards illegal drugs, seems at least biased. Intervention studies to enhance the treatment efficacy of alcohol and nicotine use disorders are urgently needed in parallel to developing and evaluating novel and effective treatment approaches for cocaine-, opiate-, amphetamine- and cannabis-related disorders.

Second, substance use disorders are not categorical (addicted vs. not addicted) but dimensional, ranging from early stages of disease where counseling or brief intervention can be offered (or sometimes even a spontaneous recovery is possible) to stages where patients may require life-long treatment and care. Drug- and rewardrelated changes in cognition, memory and motivation rather than functional adaptation and physical withdrawal represent the core of addiction, moving subjects step by step from preference to urge, from liking to wanting. Keeping this in mind, interventions should start long before chronic addiction develops and, in consequence, should aim at both risk-reduction and abstinence, contingent upon individual symptom patterns and the stage of the disease.

The recently published draft of the Diagnostic and Statistical Manual of Mental Disorders (DSM-5) of the American Psychiatric Association reflects this shift by adding additional diagnostic criteria focusing on cognitive and behavioral aspects of addiction, the inclusion of disordered gambling (and optionally internet addiction), and a first step towards a dimensional diagnosis by differentiating 'moderate' and 'severe' addiction according to the number of criteria fulfilled. It is also suggested to replace the term 'dependence' by the term 'addiction' in order to emphasize the importance of cognitive and motivational processes over signs of tolerance and withdrawal. Although no final decision has been made on this issue, a journal carrying 'addiction' in its title seems to be at the forefront of these developments.

European Addiction Research is developing rapidly too, and will become a mature and increasingly important journal. In order to enable this, the publisher and the editors decided to broaden the setup of European Addiction Research. Additional board members have been invited to strengthen the competences in addiction research ranging from neuroscience to social science and far beyond. European Addiction Research is and remains a multidisciplinary, innovative and dynamic journal, not only for strategic reasons but because it is our conviction that this is the only way to make progress. The journal will appear more frequently allowing us to display the broad progress in the field: two additional issues per year will carry the content to the scientific community bimonthly.

We would like to thank Christian Haasen for his exceptional editorial work for the journal during the last 5 years. Christian decided for his second love: therapeutic work with addicted patients. Since he will stay closely attached to the journal, he will help us not to get out of focus of what all research in the field is finally about: helping to understand and prevent addiction and improving treatment of subjects suffering from addictive behavior and its consequences.

Falk Kiefer, Mannheim Wim van den Brink, Amsterdam 